\title{
MEDICAL OPINION
}

\section{Non-alcoholic fatty liver disease, obesity and other illnesses}

\author{
Giovanni Tarantino MD
}

Department of Clinical and Experimental Medicine, Federico II University Medical School of Naples, Italy

Manuscript submitted 23rd May, 2008

Manuscript accepted 27th July, 2008

Clin Invest Med 2008; 31 (5): E290-E295.

\begin{abstract}
The estimated prevalence of NonAlcoholic Fatty Liver Disease in the general population in western countries is about $30 \%$, but it is higher among obese and diabetic people. It is likely that more sophisticated approaches are required to understand its pathogenesis and to develop drug targets. In the meantime, the range of associations between NAFLD and other illnesses broadens. Although association does not mean causation, the link between some diseases and NAFLD suggests a common mechanism.
\end{abstract}

\section{List of Abbreviations}

$\begin{array}{ll}\text { MS } & \text { Metabolic Syndrome } \\ \text { CVD } & \text { CardioVascular Disease } \\ \text { CAD } & \text { Coronary Artery Disease } \\ \text { IR } & \text { Insulin Resistance } \\ \text { NAFLD } & \text { NonAlcoholic Fatty Liver Disease } \\ \text { FL } & \text { Fatty Liver } \\ \text { AST } & \text { ASpartate aminoTransferase } \\ \text { ALT } & \text { ALanine aminoTransferase } \\ \text { GSD } & \text { GallStone Disease } \\ \text { NASH } & \text { NonAlcoholic SteatoHepatitis } \\ \text { CKD } & \text { Chronic Kidney Disease } \\ \text { PAI-1 } & \text { Plasminogen Activator Inhibitor-1 } \\ \text { CT } & \text { Computed Tomography } \\ \text { BMI } & \text { Body Mass Index } \\ \text { FMD } & \text { Flow-Mediated Dilation } \\ \text { IMT } & \text { Intima-Media Thickness } \\ \text { HPA } & \text { Hypothalamic-Pituitary-Axis } \\ \text { DHEA-S } & \text { Dehydroepiandrosterone Sulphate } \\ \text { TSST } & \text { Trier Social Stress Test }\end{array}$

DILI Drug Induced Liver Injury

25(OH)D 25-hydroxyvitamin D(3)

HOMA HOmeostasis Model Assessment

vs. versus

Engagement in sedentary employment and leisure activities has created conditions in which ancestral metabolic pathways are no longer valid. The current obesity epidemic is fuelled by the enormous availability of food and the low requirement for physical activity in our modern environment. If excess energy intake occurs over a prolonged period, most of the surplus is stored in the body as fat. Although the body has strategies that act to maintain body weight over time, they primarily defend against starvation and are less robust in preventing the development of obesity. Weight gain and increased adiposity leads to a spectrum of diseases burdened by increased health care costs. Human obesity will lead to a decrease of life expectancy for the first time in the modern era.

The liver is an important organ closely associated with lipid and glucose metabolism, whose impairment leads to the Metabolic Syndrome (MS) that is a result of Insulin Resistance (IR). NonAlcoholic Fatty Liver Disease (NAFLD) is a further expression of MS and ranges from Fatty Liver (FL) at the most clinically 
benign end of the spectrum, through an intermediate, generally progressive lesion, NonAlcoholic SteatoHepatitis (NASH), to cirrhosis.

The usual explanation for the origin of NAFLD is that increased flow of free fatty acids from visceral fat to the liver via the portal veins affects intracellular lipid metabolism. In hepatocytes, both Insulin Resistance and excess free fatty acids impair mitochondrial oxidation of fatty acids, which accumulate and contribute to the generation of free radicals by activating the metabolic pathways of peroxisomes and microsomes. ${ }^{1}$

The most accepted explanation for progression from simple FL to NASH is the "two-hit hypothesis." Over-accumulation of fat alone is insufficient to induce progression to steatohepatitis, but renders the liver more susceptible to "second hits" that, once imposed upon the steatotic liver, cause further derangement that culminates in the development of NASH. ${ }^{2}$ In this context, critical nodes of energy homeostasis play certain roles. ${ }^{3}$

\section{Importance of recognising NASH}

NASH increases the probability of developing liver cirrhosis and hepato-carcinoma. Further, NAFLD is correlated with an increased Cardio Vascular Disease (CVD) risk. ${ }^{4}$

\section{Predisposing Factors}

Periodontal Disease (PD) is associated with CVD events, suggesting that the level of systemic bacterial exposure from periodontitis could contribute to the atherosclerotic process. ${ }^{5}$ Some $5 \%$ to $20 \%$ of the population suffer from severe, generalised periodontitis, although mild to moderate periodontitis affects the majority of adults. Periodontitis results from a complex interplay of bacterial infection and host response, modified by systemic factors. The relationship between periodontitis and liver disease was studied in 172 dentulous Japanese women (20-59 years old). After multivariate adjustment for age, smoking history and oral hygiene the prevalence of periodontitis (deepest probing depth $\geq$ of $4 \mathrm{~mm}$ ) correlated with increased serum concentrations of ASpartate aminoTransferase (AST), ALanine aminoTransferase (ALT) and cholinesterase and an AST-to-ALT ratio of $<1$. Further adjustment for either Body Mass Index (BMI) or percent body fat did not change these relationships. ${ }^{6}$

These results suggest that periodontitis contributes to cause NAFLD. Further more, these patients might benefit from an intensive periodontal therapy to prevent NAFLD/CAD progression.

The classical risk factors of NAFLD, mainly obesity, do not fully explain recent epidemiological observations. Patients with schizophrenia are characterized by an increased prevalence of obesity, type 2 diabetes mellitus, and CVD. Moreover, the frequency of MS is increased in schizophrenic populations. Antipsychotic drugs may be associated with an increased risk of acute (ketoacidosis), sub acute (weight gain, IR, dyslipidemia), and chronic (diabetes, hypertension, CVD) metabolic complications. Medications might interfere with CNS function to regulate energy balance even though patients generally report an increase of appetite for sweet and fatty foods or "food craving" but also gain weight despite reduced appetite due to altered basal metabolic rate. A complex of hormones, neurotransmitters and cytokines interacting with the hypothalamus regulates appetite and eating. Among them leptin and TNF-alpha play major roles. Although the exact mechanism of antipsychotic associated weight gain is unclear, their impact on the serotonergic system and on neuropeptide $\mathrm{Y}$, a stimulator of food intake, might be implicated in the development of obesity. In addition, weight gain-related hyperleptinemia could be a link between overweight and hepatotoxicity via cytokines. Physicians should monitor body weight, liver function tests, fasting blood glucose, and lipid profile at the beginning of therapy with antipsychotic drugs. Patients complaining about weight gain should be monitored more carefully during the maintenance phase. ${ }^{7}$ 
Studies have recently suggested that NAFLD with elevated GGT concentration is associated with an increased Chronic Kidney Disease (CKD), estimated by evaluating the glomerular filtration rate and weighing proteinuria, risk among nondiabetic, nonhypertensive Korean men, irrespective of the MS presence. ${ }^{8}$

One possible mechanism could involve elevated levels of Plasminogen Activator Inhibitor-1 (PAI-1) as seen in NASH following the IR state. ${ }^{9}$ However, this interpretation conflicts with a previous study showing that PAI-1, measured in plasma from eight patients with type 1 diabetes complicated by proliferative retinopathy, and from eight patients with type 1 diabetes and background or no retinopathy, matched for age, sex and duration of diabetes, was lower in the group with proliferative retinopathy. ${ }^{10}$ Further research is required to confirm these results and to evaluate whether NAFLD contributes to the development or progression of CKD and retinopathy.

GallStone Disease (GSD) has a high prevalence among fat, fecund, middle-aged women. Conversely, in both sexes, FL predicted GSD. ${ }^{11}$ There is a strong relationship between hypomagnesemia and IR. As NASH is related to IR, the potential relationship between decreased hypomagnesemia and NASH was determined in 60 obese patients with diabetes, IR, and non-insulin resistance compared with controls. Liver biopsy was performed in subjects with elevated AST and ALT levels. NASH was diagnosed in 29 individuals who exhibited lower serum magnesium levels: those with fibrosis showed the lowest serum magnesium concentration. Multiple regression analysis adjusted for age showed that hypomagnesemia was independently related to a high HOMA (OR 7.6, CI 95\% 2.1-11.2). After adjusting for age and a high HOMA, hypomagnesemia remained independently related to NASH (OR 1.4, CI 95\% 1.1-5.4). ${ }^{12}$ Others have pointed out the protective role of magnesium consumption in the prevention of symptomatic GSD among men. ${ }^{13}$

Subclinical Cushing's syndrome caused by adrenal incidentalomas is frequently associated with over- weight and IR. Hepatic steatosis may occur in association with IR and obesity, two features commonly seen in Cushing's syndrome. Rockall et al assessed the prevalence of FL in 50 patients with active Cushing's syndrome using Computed Tomography (CT) and identified associations between hepatic steatosis, endocrine and biochemical variables and body fat distribution. In 26 patients, abdominal fat measurements were also available. Ten of 50 patients had a liver-tospleen CT attenuation ratio of $<1$, indicating hepatic steatosis. There was a positive correlation between liver-to-spleen CT attenuation ratio and alkaline phosphatase levels but with no other serum marker of Cushing's syndrome activity or liver enzyme. ${ }^{14}$

While there is general agreement on the strong association between central or visceral obesity and cardiovascular risk factors, particularly dyslipidemia and hyperinsulinemia, the relative importance of deep vs. subcutaneous abdominal fat has not been examined. ${ }^{15}$ Obesity is a major health problem with the characteristics of a pandemic. Determinants of the illness are complex and include genetic, developmental and environmental factors. Stress related neuroendocrine dysregulation and overconsumption of high caloric food are likely to contribute to this modern health threat.

Psychosocial stress, such as mental strain at work and negative recent life events in men and stress at home in women are associated with CVD. Narita et al investigated the associations between trait anxiety, IR, and atherosclerotic progression in 35 subjects without MS. The severity of trait anxiety was positively associated with HOMA and leptin-to-adiponectin ratio, and negatively associated with the percent change of brachial artery Flow-Mediated Dilation (\% FMD), sign of subclinical atherosclerosis. HOMA and leptinto-adiponectin ratio were associated with carotid Intima-Media Tickness (IMT) and negatively associated with \% FMD. These data showed the associations between trait anxiety, IR indexes and endothelial dysfunction or atherosclerotic progression. ${ }^{16}$ 
Data from five independent studies were reanalyzed in order to determine the impact of age and sex on Hypothalamic-Pituitary-Axis (HPA) responses to an acute psychosocial laboratory stress task. The sample consisted of 102 healthy subjects with 30 older adults, 41 young adults and 31 children. All participants were exposed to the Trier Social Stress Test (TSST). The stress protocol caused ACTH and total plasma cortisol responses in older and younger male and female adults as well as salivary free cortisol responses in all six age and gender groups. ${ }^{17}$ Depression is often present in obese patients. NK-cell activity and levels of cortisol and Dehydroepiandrosterone (DHEA) were reduced in patients with major depression. More advanced NAFLD, as indicated by the presence of NASH with advanced fibrosis stage, is strongly associated with low circulating DHEA-S ${ }^{18}$.

Vitamin D deficiency is a world-wide epidemic. In adults, vitamin D deficiency induces secondary hyperparathyroidism, which cause a loss of matrix and minerals, thus increasing the risk of osteoporosis and fractures. Poor mineralization of newly laid down bone matrix in adult bone results in osteomalacia. There is evidence that implicates vitamin D deficiency with an increased risk of type I diabetes, multiple sclerosis, rheumatoid arthritis, CVD, and many common deadly cancers. Measurement of 25-hydroxyvitamin D. ${ }^{3}[25(\mathrm{OH}) \mathrm{D}]$ should be part of an annual physical examination.

Marked decreases in winter serum $25(\mathrm{OH}) \mathrm{D}$ concentrations were found in 60 consecutive patients with biopsy-proven NAFLD, that was little affected by adjustment for age, sex, BMI, HOMA, and MS. Among NAFLD patients, decreased $25(\mathrm{OH}) \mathrm{D}$ concentrations were closely associated with the histological severity of hepatic steatosis, necroinflammation and fibrosis. The low concentrations of $25(\mathrm{OH}) \mathrm{D}$ found in NAFLD may mirror 'unhealthy' lifestyle (inadequate milk diet or poor sunlight exposure) or due to MS which has been found to relate to severity of hypovitaminosis D. Others have shown that vitamin D supplementation improves insulin secretion. ${ }^{19}$ Also, vitamin D defi- ciency adversely affects the activity/expression of macrophages and $\mathrm{T}$ lymphocytes, thus inducing lowgrade chronic inflammation through the increased production of proinflammatory cytokines. Low $25(\mathrm{OH}) \mathrm{D}$ can influence the migration, proliferation and gene expression of fibroblasts and vascular smooth muscle cells, and can induce fibrogenesis. ${ }^{20}$ Recent studies have reported that vitamin D-deficient individuals have increased plasma concentrations of interleukin-6. Finally, low concentrations of vitamin D result in elevations of parathyroid hormone which has been linked to insulin resistance and increases in the serum concentrations of many acute-phase proteins. ${ }^{21}$

Severe malnutrition, anemia and thrombocytopenia are strong independent predictors of mortality in HIV-infected patients. ${ }^{22}$ However, the frequency of CAD is increasing among HIV seropositive persons. This may be related to the use of antiretroviral medications and increased length of survival, or the synergism of these factors. Antiretroviral drugs can lead to patterns of injury that include "simple" steatosis and NASH. HIV-associated conditions such as hyperlactatemia and lipodystrophy frequently overlap with NAFLD. ${ }^{23}$

Hepatic mitochondrial dysfunction is likely to be crucial to the pathogenesis of NAFLD. The relatively high prevalence of Drug Induced Liver Injury (DILI) in NAFLD patients ${ }^{24}$, zeroes in on Cytochrome P-450 role. Are the "metabolic" patients genetically predisposed to NAFLD (rapid metabolizers)?

Bariatric procedures. Exercise, in conjunction with reduced caloric intake, is beneficial for weight reduction and consequently for NAFLD. There are many reasons, including increased energy expenditure, prevention of a decrease in resting energy expenditure, reduction in appetite, and prevention of muscle and bone mineral losses. But, more drastic measures are often necessary. Bariatric surgery is the main approach. Its influence on carbohydrate metabolism requires particular attention in the light of recent acquisitions suggesting metabolic improvement. Generally, 
bariatric surgery is one of the most successful technique to lose weight. ${ }^{25}$

Other types of surgery may affect NAFLD. Pancreatoduodenectomy influences hepatic fat content and is associated with hepatic steatosis. ${ }^{26}$ Diarrhoea and weight loss are common and arise from varying aetiologies, including postoperative activation of silent celiac disease. ${ }^{27}$

\section{"The real voyage of discovery consists not in making new landscapes but in having new eyes." Marcel Proust}

\section{References}

1. Hashimoto T, Fujita T, Usuda N, et al. Peroxisomal and mitochondrial fatty acid beta-oxidation in mice nullizygous for both peroxisome proliferator-activated receptor alpha and peroxisomal fatty acyl-CoA oxidase. Genotype correlation with fatty liver phenotype. J Biol Chem 1999;274:19-36.

2. Day CP, James O. Steatohepatitis: a tale of two "hits"?, Gastroenterology 1998;114:842-5

3. Tarantino G, Marra M, Contaldo F, Pasanisi F.Basal metabolic rate in morbidly obese patients with nonalcoholic fatty liver disease.Clin Invest Med. 2008;31:24-9.

4. Adibi P, Sadeghi M, Mahsa M, Rozati G, Mohseni M. Prediction of coronary atherosclerotic disease with liver transaminase level. Liver Int. 2007;27:895-900.

5. Mustapha IZ, Debrey S, Oladubu M, Ugarte R. Markers of systemic bacterial exposure in periodontal disease and cardiovascular disease risk: a systematic review and meta-analysis. J Periodontol. 2007;78:2289-302.

6. Saito T, Shimazaki Y, Koga T, Tsuzuki M, Ohshima A. Relationship between periodontitis and hepatic condition in Japanese women. J Int Acad Periodontol 2006;8:89-95.

7. Holtmann M, Kopf D, Mayer M, Bechtinger E, Schmidt MH. Risperidone-associated steatohepatitis and excessive weight-gain. Pharmacopsychiatry 2003;36:206-7.

8. Chang Y, Ryu S, Sung E, Woo HY, Oh E, Cha K, Jung E, Kim WS. Nonalcoholic fatty liver disease predicts chronic kidney disease in nonhypertensive and nondiabetic Korean men. Metabolism 2008;57:569-76.
9. Yener S, Akarsu M, Demir T, et al. Plasminogen activator inhibitor-1 and thrombin activatable fibrinolysis inhibitor levels in non-alcoholic steatohepatitis. J Endocrinol Invest 2007;30:810-9.

10. Simpson AJ, Booth NA, Moore NR, Lewis SJ, Gray RS. Circulating tissue-type plasminogen activator and plasminogen activator inhibitor type 1 in proliferative diabetic retinopathy: a pilot study. Acta Diabetol 1999;36:155-8.

11. Lonardo A, Lombardini S, Scaglioni F, et al. Fatty liver, carotid disease and gallstones: a study of agerelated associations. World J Gastroenterol 2006;12:5826-33.

12. Rodríguez-Hernández H, Gonzalez JL, RodríguezMorán M, Guerrero-Romero F. Hypomagnesemia, insulin resistance, and non-alcoholic steatohepatitis in obese subjects. Arch Med Res 2005;36:362-6.

13. Tsai CJ, Leitzmann MF, Willett WC, Giovannucci EL. Long-term effect of magnesium consumption on the risk of symptomatic gallstone disease among men. Am J Gastroenterol 2008; 103:375-82.

14. Rockall AG, Sohaib SA, Evans D, Kaltsas G, Isidori AM, Monson JP, Besser GM, Grossman AB, Reznek RH. Hepatic steatosis in Cushing's syndrome: a radiological assessment using computed tomography. Eur J Endocrinol 2003;149:543-8.

15. De Lusong MA, Labio E, Daez L, Gloria V. Nonalcoholic fatty liver disease in the Philippines: Comparable with other nations? World J Gastroenterol 2008;14:913-7.

16. Narita K, Murata T, Hamada T, et al. Associations between trait anxiety, insulin resistance, and atherosclerosis in the elderly: A pilot cross-sectional study. Psychoneuroendocrinology 2008;33:305-12.

17. Kudielka BM, Buske-Kirschbaum A, Hellhammer DH, Kirschbaum C. HPA axis responses to laboratory psychosocial stress in healthy elderly adults, younger adults, and children: impact of age and gender. Psychoneuroendocrinology 2004;29:83-98.

18. Charlton M, Angulo P, Chalasani N, et al. Low circulating levels of dehydroepiandrosterone in histologically advanced nonalcoholic fatty liver disease. Hepatology 2008;47:484-92.

19. Borissova AM, Tankova T, Kirilov G, Dakovska L, Kovacheva R. The effect of vitamin D3 on insulin secretion and peripheral insulin sensitivity in type 2 diabetic patients. Intern J Clin Pract 2003;57:258-61.

20. Veldman C, Cantorna M, DeLuca H. Expression of 1, 25 dihydroxyvitamin D3 receptor in the immune system. Arch Biochem Biophys 2000;374:334-8.

21. McCarty MF. Secondary hyperparathyroidism promotes the acute phase response - a rationale for sup- 
plemental vitamin D in prevention of vascular events in the elderly. Med Hypotheses 2005;64:1022-6

22. Johannessen A, Naman E, Ngowi BJ, et al. Predictors of mortality in HIV-infected patients starting antiretroviral therapy in a rural hospital in Tanzania. BMC Infect Dis 2008;8:52.

23. Crum-Cianflone NF. Nonalcoholic fatty liver disease: an increasingly common cause of liver disease among HIV-infected persons? AIDS Read 2007;17:513-8.

24. Tarantino G, Conca P, Basile V, et al. Prospective study of acute drug-induced liver injury in patients suffering from non-alcoho lic fatty liver disease. Hepatol Res 2007;37:410-5.

25. Gómez V, Riall TS, Gómez GA. Outcomes in bariatric surgery in the older patient population in Texas. J Surg Res 2008;147:270-5.
26. Nomura R, Ishizaki Y, Suzuki K, Kawasaki S. Development of hepatic steatosis after pancreatoduodenectomy. AJR Am J Roentgenol 2007;189:1484-8.

27. Maple JT, Pearson RK, Murray JA, Kelly DG, Lara LF, Fan AC. Silent celiac disease activated by pancreaticoduodenectomy. Dig Dis Sci 2007;52:2140-4.

Correspondence to:

Prof. Giovanni Tarantino

Department of Clinical and Experimental Medicine, Federico II University Medical School of Naples, Via S. Pansini, 580131 Naples, Italy e-mail: tarantin@unina.it 